\title{
PRIMEIRA OCORRÊNCIA DE CORAIS RUGOSOS NA FORMAÇÃO MAECURU (DEVONIANO)- BACIA DO AMAZONAS, ESTADO DO PARÁ ${ }^{1}$
}

\author{
VLADIMIR DE ARAÚJO TÁVORA* \& SUSANE RABELO DE SOUZA*
}

\begin{abstract}
FIRST OCCURENCE OF RUGOSA CORALS (CNIDARIA) INTHE MAECURU FORMATION (DEVONIAN)- AMAZONAS BASIN PARÁ STATE, BRAZIL The first record of rugosa corais in the Maecuru Formation, Devonian of the Amazonas Basin, Brazil, is described in this research. The genus Pseudochonophyllum confirms the mixture of faunas from three different paleobiogeographic realms (Eastern Americas, Malvinokaffric and Old World), suggested until now, also be brachiopods and bivalve mollusc faunas.
\end{abstract}

Keywords: Rugosa corais, Maecuru formation, Amazonas Basin, Devonian, Brazil.

RESUMO Restos de corais rugosos são registrados e descritos pela primeira vez naFormação Maecuru, Devoniano da Bacia do Amazonas. O reconhecimento do gênero Pseudochonophyllum vem corroborar a mistura de faunas dos domínios paleobiogeográficos Americas Orientais, Malvinocáfrica e Velho Mundo em tempos eodevonianos, caracterizada no Brasil, até então, somente por braquiópodes e moluscos bivalves.

Palavras-chaves: Corais rugosos, Formação Maecuru, Bacia do Amazonas, Devoniano, Brasil

INTRODUÇÃO A Formação Maecuru foi individualizada como unidade litoestratigráfica por Derby (1878), que assim denominara os sedimentitos devonianos aflorantes no Rio Maecuru. Constituída por arenitos finos a grossos intercalados com siltitos e folhelhos, possui um rico e variado conteúdo fossilífero que permite caracterizar seu ambiente deposicional como marinho de águas neríticas e deltaico (Machado 1990a, Cunha et al. 1994).

Ao contrário dos acritarcas e quitinozoários uniformemente distribuídos ao longo da unidade, os macrofósseis, preservados dominantemente sob a forma de moldes, concentram-se nas camadas mais superiores. Em seu conteúdo paleontológico figuram braquiópodes (dominantes), biválvios, gastrópodes, tentaculitídeos, trilobitas, corais, briozoários, crinóides, icnofósseis, esporos e escolecodontes, além dos citados acritarcas e quitinozoários, que posicionam temporalmente os sedimentos Maecuru entre o Emsiano e o Eo- Eifeliano (Machado, 1990a Cunha et al 1994).

A ocorrência de cnidários na Formação Maecuru foi registrada primeiramente por Katzer (1897), que individualizou duas novas espécies de corais tabulados, Chaetetes carvalhoanus e Pleurodictyum amazonicum, figuradas somente anos mais tarde pelo autor, em seu trabalho de 1903.

Posteriormente, Clarke (1913) considerou C. carvalhoanus como briozoário. Este fato foi corroborado por Oliver Jr. (1980), que lembrou ser comum na época de Katzer, confundir briozoários monticuloporídeos com corais do tipo Chaetetes. Assim, Oliver Jr. (1980) ao discutir sobre a fauna coralínea da Província Americana Oriental, considera apenas $P$. amazonicum como representante dos corais antozoários nas camadas devonianas brasileiras.

O escopo principal deste trabalho é apresentar o primeiro registro de corais rugosos nos estratos da Formação Maecuru. Trata-se de um único exemplar, procedente de um afloramento situado nas margens do Rio Maecuru, próximo da confluência com o Igarapé Ipixuna, município de Monte Alegre, noroeste do Estado do Pará (Fig.l). Este coral foi classificado como pertencente ao género Pseudochonophyllum Soshkina 1937.

PROCEDÊNCIA DO MATERIAL ESTUDADO Em 1986, geólogos da Petróleo Brasileiro S/A (PETROBRÁS) percorreram o mesmo roteiro de O. A. Derby, quando, em 1878 pela Comissão Geológica do Império, executou mapeamento geológico na Região do Baixo Amazonas. Neste trabalho de campo, denominado "Expedicão O. Derby", foram coletadas aproximadamente 5 toneladas de rochas da Formação Maecuru, ao longo do rio homónimo e adjacências. Todo esse acervo, pertencente à colecão de macrofósseis do CENPES/PETROBRÁS, está sendo utilizado como material de trabalho de um projeto de pesquisa intitulado "Paleontologia e Bioestratigrafia do Membro Lontra/ Formacão Maecuru (Devoniano Médio) da Bacia do Amazonas- Pará", coordenado pela Profa. Dra. Deusana Machado da UNI-RIO (Rio de Janeiro).
Em meio as amostras já examinadas pela equipe do projeto, foi encontrado um exemplar de coral da Ordem RUGOSA, ainda não registrado no Devoniano do norte do Brasil.

O espécimen registra, em de moldes, as feições externas e internas do organismo, em um arenito fino, ferruginoso, e rico em outros organismos, como braquiópodes, biválvios, trilobitas, briozoários e crinóides.

SISTEMÁTICA PALEONTOLÓGICA Utilizam-se no presente estudo, as classificações sistemáticas adotadas por Pedder \& Feist (1998) e Hill (1956). A nomenclatura morfológica e os termos aplicados nas descrições sistemáticas, seguem a proposta original de Moore et al. (1956), ainda usada atualmente.

Pkyllum CNIDARIA Hatschek 1888

Classis ANTHOZOA Ehrenberg 1834

Ordo RUGOSA Milne-Edwards \& Haime 1850

Sub Ordo PTENOPHYLLINA Wedekind 1927

Família SPONGOPHYLLIDAE Dybowski 1873

Sub Família PTENOPHYLLINAE Wedekind 1923

Diagnose Espongofilídeos com coralitos de tamanho médio a grande, com marginaria ampla. Cálice profundo, com base bem abaixo da borda superior das partes periaxiais dos septos maiores. Septos trabeculados no marginarium; trabéculas subtilmente alinhadas, formando flanges; possivel ocorrência de vepréculas na parte interna do dissepimentário e porção superior do tabularium. Dissepimentos mais numerosos e marginando todo o tabularium em alguns géneros e espécies. Tabulas tipicamente interrompidas pelos septos maiores e seus flanges, rigorosamente espacadas em todos os géneros. Fóssula indistinta (traduzido de Pedder \& Fest 1998, p.985).

\section{Genus Pseudochonophyllum Soshkina 1937}

Diagnose Coral solitário com septos naóticos e numerosos dissepimentos estreitos, regulares e globosos (traduzido de Hill 1956, p.F306).

\section{Pseudochonophyllum sp.- Figuras 2 e 3.}

Descrição Corallum trocóide. Estruturas de rejuvenescimento (estágios de crescimento do coralium) reconhecíveis no marginarium, consistindo em intervalos regulares que apresentam bandas amplas onde ficam visíveis os septos do cálice anterior. Plataforma calicular ampla. Cálice profundo e expandido. Tabulas retilíneas, entumescidas e regularmente espacadas. Septos retilíneos, espessos, arredondados, bilateralmente arranjados, com bordos em ziguezague, mais largos exteriormente, caracterizando uma estereozona incompleta. Septos de segunda ordem intercalados com os de primeira ordem, visíveis apenas no tabularium. Septos naóticos nas porções externa e média do marginarium. Partes naóticas dos septos consistindo em placas pequenas, subhorizontais e arqueadas, alinhadas no maior comprimento dos 

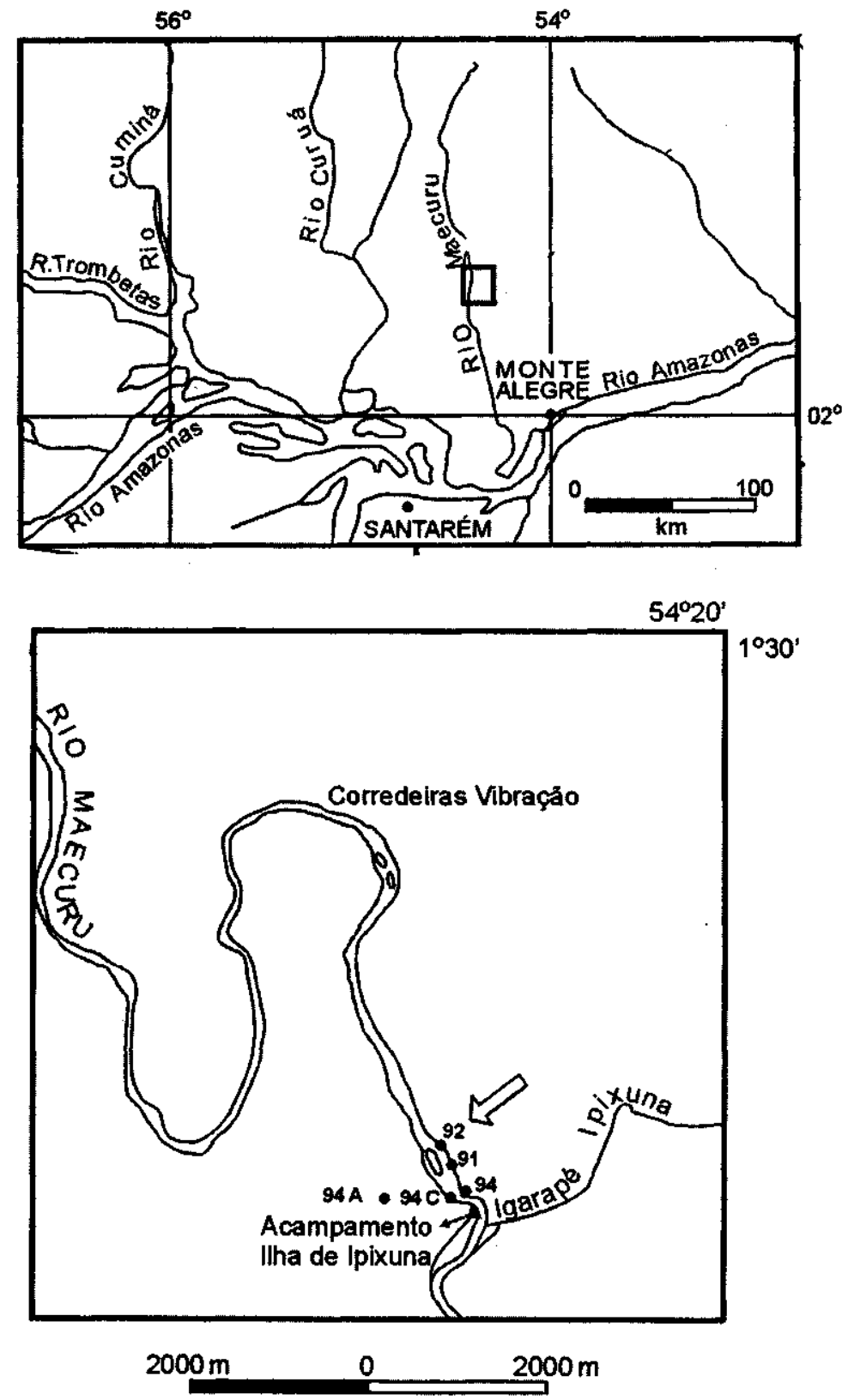

Figura l-Mapa de localização do afloramento em que foi realizada a coleta.

septos. Trabécula não preservada. Dissepimentos pequenos, globosos, estreitos, interpostos e perpendiculares aos septos, definindo uma zona marginal no cálice, o dissepimentário.

Medidas diâmetro maior do corallum: $8.8 \mathrm{~mm}$

altura do corallum: $14.2 \mathrm{~mm}$

maior comprimento dos septos: $6 \mathrm{~mm}$

espessura dos septos: $0.2 \mathrm{~mm}$

espessura das zonas dissepimentárias entre os septos: $0.1 \mathrm{~mm}$

espessura das tabulas: $1.5 \mathrm{~mm}$

espaço entre as tabulas: $1 \mathrm{~mm}$

diâmetro da plataforma calicular: $2.1 \mathrm{~mm}$

MATERIAL Um exemplar preservado na forma de impressões (molde interno e externo), catalogado como FM92-1063 LE, procedente do ponto 92, afloramento da Formação Maecuru, situado nas margens do Rio Maecuru, próximo da confluência com o Igarapé Ipixuna(Fig. 1).

DISCUSSÃO Segundo Hill (1956) para se efetivar classificação sistemática de corais rugosos, é necessária a confecção de lâminas delgadas, para observação de suas estruturas internas. Entretanto, os autores do presente trabalho concordam com Clarkson (1986, p.95), que o estudo de microestruturas dos corais rugosos não está tão desenvolvido a ponto de ter muita utilidade. Em seção fina se conhece apenas um pouco mais sobre o dissepimentário, zona marginal perfeitamente reconhecida sob exame em lupa binocular de alta resolução. Para nós, este grupo sistemático não apresenta homomorfia externa tão marcante quanto nos briozoários paleozóicos (criptostomados, cistoporados e trepostomados).

O exemplar descrito guarda as feições morfológicas típicas do género Pseudochonophyllwn, tais como os septos naóticos e a ornamentação dos septos de primeira ordem no marginarium, os dissepi- 


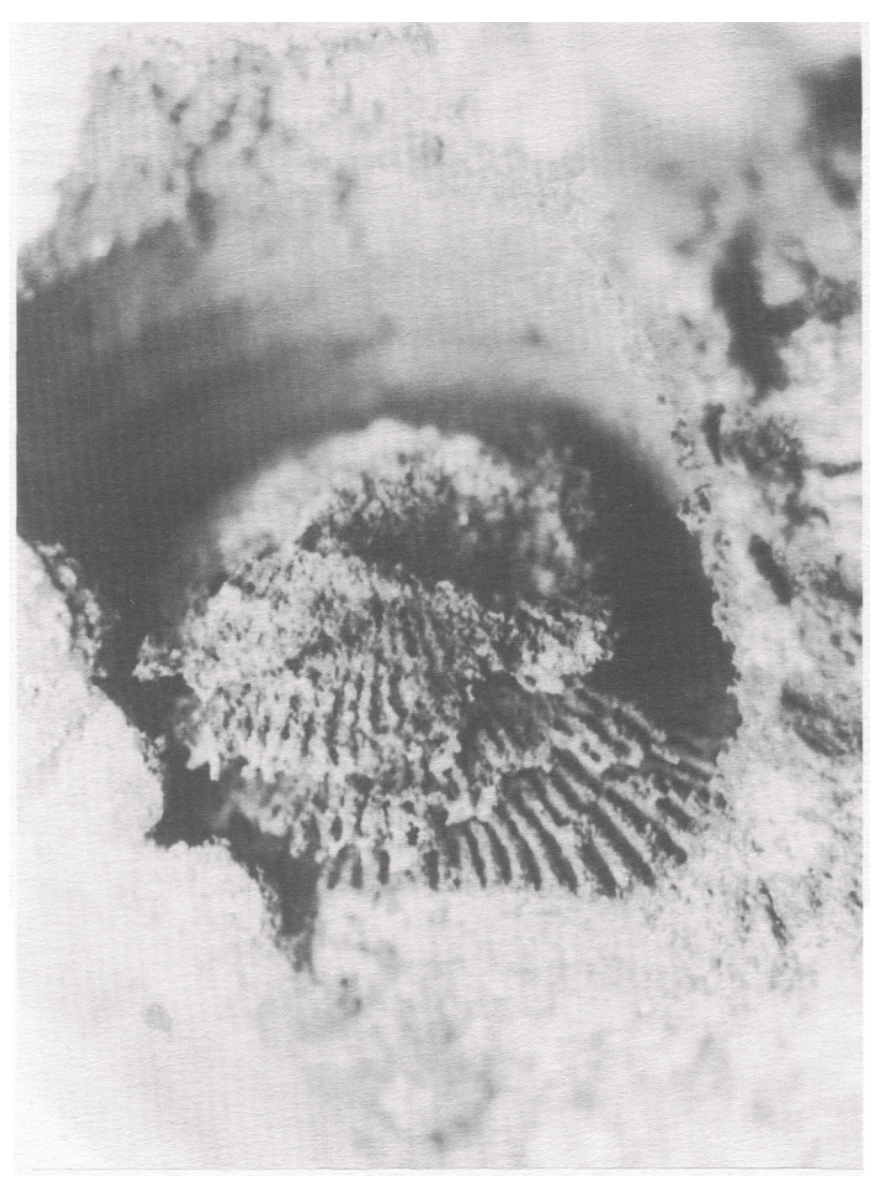

Figura 2 - Detalhe do molde interno mostrando os septos de primeira e segunda ordem, dissepimentos e plataforma calicular (X3.7).

mentos. A afinidade com a espécie $P$. sentum está representada pelo caráter amplo da plataforma calicular e pelas pequenas, subhorizontais e arqueadas placas, reconhecidas nas partes naóticas dos septos de primeira ordem.

Entretanto, a não preservação do septo periaxial e da trabécula impediu aos autores considerar o fóssil da Formação Maecuru como representante da espécie reconhecida na Formação Izarne (França). Da mesma forma, a fragmentação da parte superior do corallum, que não permitiu visualizar claramente os limites entre dissepimentário e tabularium, contribuiu para a sua classificação sistemática ficar indeterminada a nível específico.

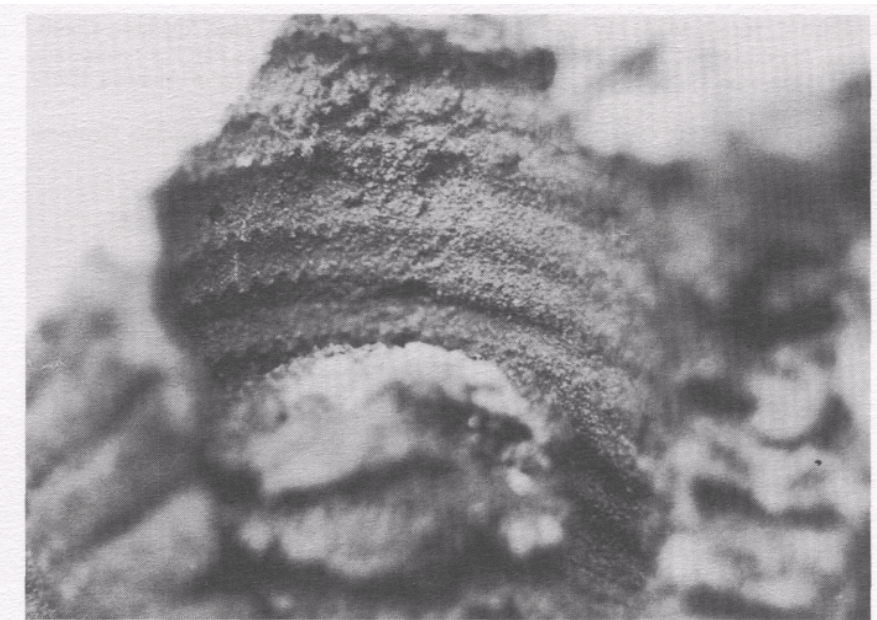

Figura 3 - Detalhe do molde externo mostrando as tabulas e as terminações dos septos em ziguezague (X3.3).

CONCLUSÕES A mistura de faunas "austrais" e "boreais" na Formação Maecuru foi constatada pela primeira vez por Katzer (1 897). Loczy (1966), Harrington (1967), Melo (1985, 1988), Boucot (1975, 1988) e Machado (1990a e b), também atestaram esta mescla, identificando nestes sedimentitos, espécies debraquiópodes e biválvios afins àquelas dos domínios paleobiogeográficos Velho Mundo, Américas Orientais e Malvinocáfrica.

Pedder (in Pedder \& Fest 1998) reconheceu a espécie nova Pseudochonophyllum sentum na Formação Izarne (sul da França), datada como emsiana, idade coincidente em parte, com a Formação Maecuru.

Com este trabalho, estende-se a afinidade das faunas marinhas emsianas de braquiópodes e biválvios do Brasil, América do Norte e algumas regiões europeias também para os corais rugosos, pela ampliação da área de ocorrência do género Pseudochonophyllum, antes restrito a algumas camadas devonianas da Europa. O reconhecimento deste género de coral rugoso no Devoniano da Bacia do Amazonas, constitui assim um importante indicador paleobiogeográfico .

Assim, com base nas considerações supracitadas e na notável afinidade morfológica do exemplar aqui descrito com a espécie $P$. sentum, considera-se que o espécimen encontrado na Formação Maecuru seja um representante do género Pseudochonophyllum. A classificação sistemática em aberto a nível específico é justificada pelo estado de preservação do exemplar disponível, que guarda indubitavelmente apenas as feições morfológicas genéricas. O progresso das pesquisas no projeto ao qual se vinculam os autores, poderá fornecer outros espécimens melhor preservados, que permitam caracterização sistemática mais detalhada.

\section{Referências}

Boucot A.J. 1975. Evolution and Extinction Rate Controls. Developments in Paleontology and Stratigraphy 1, Amsterdam, Elsevier, xv, 427p.

Boucot A.J. 1988. Devonian Biogeography: an Update. In: Canadian Society of Petroleum Geologists, International Symposium on the Devonian System, 2, Calgary, Proceedings, 3:211-227.

Clarke J.M. 1913. Fósseis Devonianos do Paraná. Monografia do Serviço Geológico e Mineralógico do Brasil, 1:1-353.

CLARKSON, E.N.K. 1986. Cnidarios. In: E.N.K. Clarkson (ed.) Paleontologia de Invertebrados y Su Evolución. Madrid, Editorial Paraninfo S.A., 77-113.

Cunha P.R.C., Gonzaga F.G., Coutinho L.F.C., Feijó F.J. 1994. Bacia do Amazonas. Boletim de Geociências da Petrohrás, 8:47-55.

Derby O. A. 1878. Contribuições para a Geologia da Região do Baixo Amazonas. Archivos do Museu Nacional, 2:77-104.

Harrington H.J. 1967. Devonian of South America. In: Alberta Society of Petroleum Geologists, International Symposium on the Devonian System, 1, Calgary, Proceedings, 1:651-671.

Hill D. 1956. RUGOSA. In: Moore, R.C. (ed.) Treatise on Invertebrate Paleontology, Part F, Coelenterata, Lawrence, University of Kansas Press and Geological Society of America, F233-F324.
Katzer F. 1897. A Fauna Devonica do Rio Maecuru e as Suas Relações com a Fauna de Outros Terrenos Devonicos do Globo. Boletim do Museu de História Natural e Ethnografia, 2:204-246.

Katzer F. 1903. Grundzüge der Geologie des Unteren Amazonsgebeites (dês Staates Pará in Brasilien). Leipzig, Max Weg, 298p. (traduzido em 1933- Geologia do Estado do Pará (Brasil). Boletim do Museu Paraense Emílio Goeldi, 9:1-270).

Loczy L. 1966. Contribuições à Paleogeografiae Historiado Desenvolvimento Geológico da Bacia do Amazonas. Boletim da Divisão de Geologia e Mineralogia, 223:1-96.

Machado D.M.C. 1990a. Biválvios Devonianos da Bacia do Amazonas (formações Maecuru e Ererê): Considerações Sistemáticas e Paleoautoecológicas. Instituto de Geociências, Universidade Federal do Rio de Janeiro, Dissertação de Mestrado, 285p.

Machado D.M.C. 1990b. Algumas Considerações Estratigráficas e Biogeográficas Acerca dos Biválvios Devonianos da Bacia do Amazonas. In: SBG- Núcleo Nordeste, Congresso Brasileiro de Geologia, 36, Natal, Anais, 1:425-435.

Melo J.H.G. 1985. A Província Malvinocáfrica no Devoniano do Brasil: Estado Atual dos Conhecimentos. Instituto de Geociências, Universidade Federal do Rio de Janeiro, Dissertação de Mestrado, 890p. 
Melo J.H.G. 1988. The Malvinokaffric Realm in the Devonian of Brazil. In: Canadian Society of Petroleum Geologists, International Symposium on the Devonian System, 2, Calgary, Proceedings, 1:669-703.

Moore R.C.Hill D., Wells J.W. 1956. Glossary of Morphological Terms Applied to Corais. In: Moore, R.C. (ed.) Treatise on Invertebrate Paleontology, Part F, Coelenterata, Lawrence, University of Kansas Press and Geological Society of America, F245Oliver Jr. W.A. 1980. Corais in the Malvinokaffric Realm. Münster Forsch. Geologie Palaontologie, 52:13-17.
PedderA.E.H. \&FeistR. 1998.Lower Devonian (Emsian) Rugosa of the Izarne Formation, Montagne Noire, France. Journal of Paleontology, 72:967-991.

.. A 1079

Recebido em 14 de marco de 1999 Recebido em 14 de marco de
Revisão dos autores em 20 de agosto de 1999 Revisão aceita em 22 de agosto de 1999 